\title{
LÉVY MEASURES FOR A CLASS OF MARKOV SEMIGROUPS IN ONE DIMENSION
}

\begin{abstract}
BY
KEN-ITI SATO $\left.{ }^{1}\right)$

Abstract. Given a Markov semigroup of linear operators in the space of realvalued continuous functions on the line vanishing at infinity, we prove that the Lévy measure exists if the domain of the infinitesimal generator contains $\mathfrak{D}_{K}\left(D_{m} D_{s}^{+}\right)$, the domain of William Feller's generalized second order differential operator restricted to functions with compact supports. We give estimate of singularity of the Lévy measure and representation of the infinitesimal generator. Conversely, given Lévy measure or the form of infinitesimal generator, existence of the corresponding Markov semigroup is shown under some conditions. The case of circles is also discussed.
\end{abstract}

1. Introduction. Let $C_{0}(R)$ be the Banach space of real-valued continuous functions on the real line $R$ vanishing at infinity with norm $\|f\|=\max _{x \in R}|f(x)|$, and let $C_{K}(R)$ be the subset of $C_{0}(R)$ of functions with compact supports. A family of linear operators $\left\{T_{t} ; t \geqq 0\right\}$ on $C_{0}(R)$ is called Markov semigroup or $M$-semigroup if it is a strongly continuous semigroup and if each $T_{t}$ is nonnegative with norm $\leqq 1$. A family of measures $\left\{n_{x}(d y) ; x \in R\right\}$ is called Lévy measure for $\left\{T_{t}\right\}$ if $n_{x}$ is a measure on $R \backslash\{x\}\left({ }^{2}\right)$ finite for compact sets in $R \backslash\{x\}$ and if, for each $x \in R$ and $f \in C_{K}(R)$ such that $x \notin S(f)\left({ }^{3}\right)$, we have

$$
\lim _{t \rightarrow 0+} t^{-1}\left(T_{t} f\right)(x)=\int_{R \backslash\{x\}} f(y) n_{x}(d y) .
$$

Given an $M$-semigroup, the Lévy measure is unique if it exists. In this paper we will, under the assumption that the domain $\mathscr{D}((\xi))$ of the infinitesimal generator (5) of $\left\{T_{t}\right\}$ contains $\mathfrak{D}_{K}\left(D_{m} D_{s}^{+}\right)$for a generalized second order differential operator $D_{m} D_{s}^{+}$of Feller $[2]\left({ }^{4}\right)$, prove the existence of the Lévy measure and make an

Received by the editors March 10, 1969.

AMS Subject Classifications. Primary 6062; Secondary 6060, 4748, 4750, 4770.

Key Words and Phrases. Lévy measure, Markov semigroup, Markov process, infinitesimal generator, generalized second order differential operator, dispersive operator, Cauchy process, perturbation of semigroup generators.

${ }^{(1)}$ Currently visiting University of Illinois. Supported in part by National Science Foundation at University of Minnesota and University of Illinois.

( $\left.{ }^{2}\right) E \backslash F$ is the intersection of $E$ and the complement of $F$.

$\left({ }^{3}\right) S(f)$ is the support of $f$, i.e., the closure of the set $\{x ; f(x) \neq 0\}$.

( $\left.{ }^{4}\right) D_{m} D_{s}^{+}$is $D_{v} D_{u}$ in [2] where $s(x)=u(x)$ and $m\left(x_{1}, x_{2}\right]=v\left(x_{2}\right)-v\left(x_{1}\right)$. For rigorous definition, see $\S 2$.

Copyright (C) 1970, American Mathematical Society 
estimation of its singularity. Here $\mathfrak{D}_{K}\left(D_{m} D_{s}^{+}\right)$is the set of functions with compact supports in the domain of $D_{m} D_{s}^{+}$. Let $\Gamma$ (possibly void) be the set of discontinuity points for $m$. Let

$$
\begin{gathered}
\varphi_{x}(y)=\int_{x}^{y} s(d z) m(x, z] \text { for } y \geqq x, \quad \int_{y}^{x} s(d z) m(z, x] \text { for } y<x, \\
s_{x}(y)=s(y)-s(x) .
\end{gathered}
$$

It will be proved that $n_{x}$ is finite outside a neighborhood of $x$ and that the singularity of $n_{x}$ at $x$ is such that $\varphi_{x}(y)$ or $\left|s_{x}(y)\right|$ is integrable with respect to $n_{x}$ in a neighborhood of $x$ according as $x \in R \backslash \Gamma$ or $x \in \Gamma$ (§3). Using this, we will get representation of $\& f$ for $f \in \mathfrak{D}_{K}\left(D_{m} D_{s}^{+}\right)$in the following integro-differential form $(\S 4)$ :

$$
\begin{aligned}
\text { as } f(x)= & a(x) D_{m} D_{s}^{+} f(x)+b(x) D_{s} f(x)+c(x) f(x) \\
& +\int_{R \backslash\{x\}}\left[f(y)-f(x)-\chi_{U}(y) s_{x}(y) D_{s} f(x)\right] n_{x}(d y)
\end{aligned}
$$

for $x \in R \backslash \Gamma$ where

$$
a(x) \geqq 0, \quad c(x) \leqq 0
$$

and $U$ is a bounded open interval containing $x\left({ }^{5}\right)$, and

$$
\begin{aligned}
\text { Bf } f(x)= & (m\{x\})^{-1}\left(a^{+}(x) D_{s}^{+} f(x)-a^{-}(x) D_{s}^{-} f(x)\right)+c(x) f(x) \\
& +\int_{R \backslash\{x\}}[f(y)-f(x)] n_{x}(d y)
\end{aligned}
$$

for $x \in \Gamma$ where

$$
a^{+}(x) \geqq 0, \quad a^{-}(x) \geqq 0, \quad c(x) \leqq 0 .
$$

It is known that the transition semigroups of processes continuous in probability with stationary independent increments are $M$-semigroups in $C_{0}(R)$, that the domains of their infinitesimal generators (S) contain all $C^{2}$ functions with compact supports, and that they have (translation invariant) Lévy measures $n_{x}(d y)$, which are finite outside a neighborhood of $x$ and make $(y-x)^{2}$ integrable near $x$. (s) has the form

$$
\begin{aligned}
\text { Sf } f(x)= & a \frac{d^{2} f}{d x^{2}}(x)+b \frac{d f}{d x}(x)+c f(x) \\
& +\int_{R \backslash\{x\}}\left[f(y)-f(x)-\chi_{(x-1, x+1)}(y) \frac{d f}{d x}(x)\right] n_{x}(d y)
\end{aligned}
$$

where $a \geqq 0, c \leqq 0$ and $b$ are constants. Our results are extension of these facts. In case all $C^{2}$ (or $C^{\infty}$ ) functions with compact supports belong to $\mathscr{D}(\mathbb{S})$, the results are already known for $R^{n}$ [1], [12], [13], [15].

$\left.{ }^{5}\right) \chi_{U}$ is the indicator function of $U$. 
Let us call an operator $A$ in $C_{0}(R)$ dispersive, if, whenever $f \in \mathfrak{D}(A)$ attains its positive maximum at $x_{0}, A f\left(x_{0}\right)$ is nonpositive. Dispersiveness is a necessary condition for an operator to generate an $M$-semigroup $\left({ }^{6}\right)$. An operator defined by the right-hand sides of (1.4) and (1.6) on $R \backslash \Gamma$ and $\Gamma$, respectively, is dispersive if the coefficients satisfy the sign conditions (1.5) and (1.7). Although we can prove the representation (1.4) for all $x \in R$ including $\Gamma$ with $D_{s}^{+} f$ or $D_{s}^{-} f$ replacing $D_{s} f$, the condition (1.5) for points in $\Gamma$ does not guarantee dispersiveness of an operator defined by the right-hand side. This is the reason why we ought to use (1.6) on $\Gamma$.

In $\$ \$ 3$ and 4 we investigate Lévy measures and infinitesimal generators, given $M$-semigroups. Turning to the converse direction, we will give in $\S 5$ sufficient conditions for operators of the form (1.4)-(1.7) to generate $M$-semigroups in $C_{0}(R)$ by using perturbation theory for semigroup generators. The $M$-semigroup thus generated has Lévy measure equal to the given $n_{x}$. In case $m$ is continuous and $D_{s}$ and $D_{m}$ are ordinary differentiation with smooth coefficients, related results are found in [6], [10].

In $\S 6$ we will show that all the results can be carried over to the case of circles from the real line.

To every $M$-semigroup in $C_{0}(R)$ there corresponds a Markov process on the line and the probabilistic meaning of the Lévy measure is investigated in [5], [6], [8], [14]. The problem of finding Markov processes which have Lévy measures equal to (or greater than) a certain given measure has a special importance connected with the study of behavior near boundary for Markov processes. Thus Motoo [9] essentially proves that in order to find all those Markov processes on the closed disk which have continuous trajectories, stay on the boundary only for a set of times of Lebesgue measure zero, and behave in the interior in the same manner as the Brownian motion, it is necessary and sufficient to find all the Markov processes on the boundary whose Lévy measures are identical with that of the Cauchy process wound around on it. It is for this reason that, in examples, we are especially interested in the existence of those $M$-semigroups which have Lévy measure $n_{x}(d y)=\pi^{-1}(y-x)^{-2} d y$ (i.e., that of the Cauchy process) in case of the line or $n_{x}(d y)=(2 \pi)^{-1}(1-\cos (y-x))^{-1} d y$ (i.e., that of the Cauchy process wound around) in case of the unit circle.

Motoo (unpublished) introduced integro-differential operators of the form (1.4) in 1966 and found the result stated in Example 6.1 with (6.3) replaced by a stronger condition

$$
\lim _{\delta \rightarrow 0+} \sup _{x \in S} \int_{\left\{y ; \theta_{x}(y)<\delta\right\}}\left|s_{x}(y)\right| m(x, y) n_{x}(d y)=0 .
$$

Our Theorem 6.4 is merely an extension of this result of his.

$\left(^{6}\right)$ We say that $A$ generates an $M$-semigroup if $A$ is its infinitesimal generator. 
2. Lemmas concerning $D_{m} D_{s}^{+}$. Suppose we are given a strictly increasing continuous function $s(x)$ on $R$ and a measure $m$ on $R$ finite for compact sets and positive for nonempty open sets $\left({ }^{7}\right)$. $s$ also induces a measure, which we denote by the same letter. Functions $\varphi_{x}(y)$ and $s_{x}(y)$ are defined by (1.2) and (1.3) and $\psi_{x}(y)$ is defined by

$$
\psi_{x}(y)=\int_{x}^{y} s(d z) m[x, z] \quad \text { for } y \geqq x, \quad \int_{y}^{x} s(d z) m(z, x) \quad \text { for } y<x .
$$

Obviously, $\varphi_{x}=\psi_{x}$ if $x \notin \Gamma$ where $\Gamma$ is the set of discontinuity points for $m$. For any open set $U$, let $\mathfrak{D}\left(D_{m} D_{s}^{+} ; U\right)$ be the set of functions $f$ on $U$ such that

(i) $f$ is continuous;

(ii) $D_{s}^{+} f(x)=\lim _{h \rightarrow 0+}((f(x+h)-f(x)) /(s(x+h)-s(x)))$ exists and is right continuous with bounded variation on any compact subset of $U$;

(iii) the induced signed measure $D_{s}^{+} f(d x)$ on $U$ is absolutely continuous with respect to $m$ and its Radon-Nikodym derivative has a continuous version, which we denote by $D_{m} D_{s}^{+} f$.

Note that $f \in \mathfrak{D}\left(D_{m} D_{s}^{+} ; U_{1} \cup U_{2}\right)$ if $[f]_{U_{1}} \in \mathscr{D}\left(D_{m} D_{s}^{+} ; U_{1}\right)$ and $[f]_{U_{2}} \in$ $\mathfrak{D}\left(D_{m} D_{s}^{+} ; U_{2}\right)\left({ }^{8}\right)$. We denote by $\mathfrak{D}_{0}\left(D_{m} D_{s}^{+}\right)$the set of $f$ such that $f \in C_{0}(R)$ $\cap \mathfrak{D}\left(D_{m} D_{s}^{+} ; R\right)$ and $D_{m} D_{s}^{+} f \in C_{0}(R)$, and by $\mathfrak{D}_{K}\left(D_{m} D_{s}^{+}\right)$the set $C_{K}(R) \cap$ $\mathfrak{D}\left(D_{m} D_{s}^{+} ; R\right)$.

We also use the left derivative $D_{s}^{-} f(x)$, the limit of $(f(x)-f(x-h)) /(s(x)-s(x-h))$ as $h \rightarrow 0+$. If $D_{s}^{+} f(x)=D_{s}^{-} f(x)$, we write the value as $D_{s} f(x)$.

We will prove some lemmas.

Lemma 2.1. Let $f$ be continuous on $\left[x_{1}, x_{2}\right]$ and let $D_{s}^{+} f$ exist in $\left(x_{1}, x_{2}\right)$. Then

$$
D_{s}^{+} f\left(x_{0}\right) \leqq \frac{f\left(x_{2}\right)-f\left(x_{1}\right)}{s\left(x_{2}\right)-s\left(x_{1}\right)} \leqq D_{s}^{+} f\left(x_{0}^{\prime}\right)
$$

for some $x_{0}$ and $x_{0}^{\prime}$ in $\left(x_{1}, x_{2}\right)$.

Proof is easy and omitted. One consequence is that if $D_{s}^{+} f(x-)$, the limit of $D_{s}^{+} f(x-h)$ as $h \rightarrow 0+$, exists, then $D_{s}^{-} f(x)=D_{s}^{+} f(x-)$. Another consequence is that if $f$ is continuous in $\left(x_{1}, x_{2}\right)$ and $D_{s}^{+} f=0$ in $\left(x_{1}, x_{2}\right)$, then $f$ is constant. More generally, we have

LEMMA 2.2. Let $f \in \mathfrak{D}\left(D_{m} D_{s}^{+} ;\left(x_{1}, x_{2}\right)\right)$ and $D_{m} D_{s}^{+} f=0$. If $f\left(x_{0}\right)=D_{s}^{+} f\left(x_{0}\right)=0$ at some $x_{0} \in\left(x_{1}, x_{2}\right)$, or $f\left(x_{1}+\right)=D_{s}^{+} f\left(x_{1}+\right)=0$, or $f\left(x_{2}-\right)=D_{s}^{+} f\left(x_{2}-\right)=0$, then $f=0$.

Proof is immediate, since we get $D_{s}^{+} f=0$.

(7) We sometimes write $m E$ instead of $m(E)$ for a set $E \subset R$.

$\left(^{8}\right)[f]_{U}$ stands for the restriction of $f$ to $U$. 
Lemma 2.3. Let $x_{1}<x_{0}<x_{2}$ and let $g$ be continuous on $\left(x_{1}, x_{2}\right)$. If

$$
\begin{array}{ll}
f(x)=a+b s_{x_{0}}(x)+\int_{x_{0}}^{x} s(d y) \int_{\left(x_{0}, y\right]} g(z) m(d z), & x \geqq x_{0}, \\
f(x)=a+b s_{x_{0}}(x)+\int_{x}^{x_{0}} s(d y) \int_{\left(y, x_{0}\right]} g(z) m(d z), & x<x_{0},
\end{array}
$$

then, we have $f \in \mathscr{D}\left(D_{m} D_{s}^{+} ;\left(x_{1}, x_{2}\right)\right)$ and

$$
f\left(x_{0}\right)=a, \quad D_{s}^{+} f\left(x_{0}\right)=b, \quad D_{m} D_{s}^{+} f=g .
$$

Similarly, if

$$
\begin{array}{ll}
f(x)=a+b s_{x_{0}}(x)+\int_{x_{0}}^{x} s(d y) \int_{\left[x_{0}, y\right]} g(z) m(d z), & x \geqq x_{0}, \\
f(x)=a+b s_{x_{0}}(x)+\int_{x}^{x_{0}} s(d y) \int_{\left(y, x_{0}\right)} g(z) m(d z), & x<x_{0},
\end{array}
$$

then, $f \in \mathfrak{D}\left(D_{m} D_{s}^{+} ;\left(x_{1}, x_{2}\right)\right)$ and

$$
f\left(x_{0}\right)=a, \quad D_{s}^{-} f\left(x_{0}\right)=b, \quad D_{m} D_{s}^{+} f=g .
$$

Conversely, every $f \in \mathfrak{D}\left(D_{m} D_{s}^{+} ;\left(x_{1}, x_{2}\right)\right)$ has the expressions (2.2) and (2.4) where $a, b, g$ are determined by (2.3) and (2.5), respectively.

Proof. It is easy to check $f \in \mathfrak{D}\left(D_{m} D_{s}^{+} ;\left(x_{1}, x_{2}\right)\right),(2.3)$, and (2.5). In order to get the converse part, we have only to note Lemma 2.2 .

RemarK 2.1. The above lemma is also true for $x_{1}=x_{0}<x_{2}$ or $x_{1}<x_{0}=x_{2}$ under suitable modification. Thus, if $f \in \mathscr{D}\left(D_{m} D_{s}^{+} ;\left(x_{1}, x_{2}\right)\right)$ and if $D_{m} D_{s}^{+} f\left(x_{1}+\right)$ $\operatorname{exists}\left({ }^{9}\right)$, then

$$
f(x)=a+b s_{x_{1}}(x)+\int_{x_{1}}^{x} s(d y) \int_{\left(x_{1}, y\right]} g(z) m(d z)
$$

where $f\left(x_{1}+\right)=a, D_{s}^{+} f\left(x_{1}+\right)=b$, and $D_{m} D_{s}^{+} f=g$. (Existence of $a$ and $b$ is proved from Lemma 2.3.)

The following three lemmas deal with some extension of functions in $\mathfrak{D}\left(D_{m} D_{s}^{+} ; U\right)$ to a wider domain $U^{\prime} \supset U$.

LEMMA 2.4. Let $x_{1}<x_{2}<x_{3}<x_{4}$ and let $f$ be a function on $\left(x_{1}, x_{2}\right) \cup\left(x_{3}, x_{4}\right)$ which is constant in each interval: $f=a$ on $\left(x_{1}, x_{2}\right), f=a^{\prime}$ on $\left(x_{3}, x_{4}\right)$. Then, $f$ can be extended to a function $\tilde{f} \in \mathfrak{D}\left(D_{m} D_{s}^{+} ;\left(x_{1}, x_{4}\right)\right)$ such that $a \wedge a^{\prime} \leqq \tilde{f} \leqq a \vee a^{\prime}\left({ }^{10}\right)$.

Proof. Suppose $a<a^{\prime}$. Let $g \in C\left[x_{2}, x_{3}\right], g\left(x_{2}\right)=g\left(x_{3}\right)=0$. Define $f$ by $\tilde{f}=f$ on $\left(x_{1}, x_{2}\right) \cup\left(x_{3}, x_{4}\right)$ and

$$
\tilde{f}(x)=a+\int_{x_{2}}^{x} s(d y) \int_{\left(x_{2}, z\right]} g(z) m(d z) \text { for } x \in\left[x_{2}, x_{3}\right] .
$$

$\left({ }^{9}\right)$ We use the word existence of a limit letting its finiteness be included.

${ }^{\left({ }^{10}\right)} a \wedge a^{\prime}$ is the smaller of $a$ and $a^{\prime}$ while $a \vee a^{\prime}$ is the larger. 
If $\tilde{f}$ satisfies $\tilde{f}\left(x_{3}\right)=a^{\prime}$ and $D_{s}^{+} \tilde{f}\left(x_{3}-\right)=0$, then $\tilde{f}$ belongs to $\mathscr{D}\left(D_{m} D_{s}^{+} ;\left(x_{1}, x_{4}\right)\right)$. Fix $x_{0} \in\left(x_{2}, x_{3}\right)$ and let $g_{1}, g_{2} \in C\left[x_{2}, x_{3}\right]$ be such that $g_{1}=0$ at $x_{2},>0$ on $\left(x_{2}, x_{0}\right),=0$ on $\left[x_{0}, x_{3}\right]$ and $g_{2}=0$ on $\left[x_{2}, x_{0}\right],>0$ on $\left(x_{0}, x_{3}\right),=0$ at $x_{3}$. We can choose positive constants $c_{1}$ and $c_{2}$ in such a way that $g=c_{2}\left(g_{1}-c_{1} g_{2}\right)$ satisfies $\int_{\left(x_{2}, x_{3}\right]} g(y) m(d y)=0$ and $\int_{x_{2}}^{x_{3}} s(d y) \int_{\left(x_{2}, y\right]} g(z) m(d z)=a^{\prime}-a$. If we use this $g, \tilde{f}$ satisfies all the requirements. The case $a=a^{\prime}$ is trivial and the case $a>a^{\prime}$ is similar.

REMARK 22 . In the above lemma, $\tilde{f}$ can be chosen to satisfy $\left\|D_{m} D_{s}^{+} \tilde{f}\right\| \leqq k\left|a-a^{\prime}\right|$, where $k$ is a constant depending on $x_{2}$ and $x_{3}$. This is clear from the proof.

LEMmA 2.5. Let $x_{1}<x_{2}<x_{3}<x_{4}$ and let $f \in \mathfrak{D}\left(D_{m} D_{s}^{+} ;\left(x_{1}, x_{2}\right) \cup\left(x_{3}, x_{4}\right)\right)$. Let $D_{m} D_{s}^{+} f\left(x_{2}-\right)$ and $D_{m} D_{s}^{+} f\left(x_{3}+\right)$ exist. Then, for any $\varepsilon>0$ we can find $\tilde{f} \in$ $\mathcal{P}\left(D_{m} D_{s}^{+} ;\left(x_{1}, x_{4}\right)\right)$ which is an extension of $f$ and satisfies

$$
\left(a \wedge a^{\prime}\right)-\varepsilon<\tilde{f}<\left(a \vee a^{\prime}\right)+\varepsilon
$$

on $\left[x_{2}, x_{3}\right]$ where $a=f\left(x_{2}-\right)$ and $a^{\prime}=f\left(x_{3}+\right)$.

Proof. Let $b=D_{s}^{+} f\left(x_{2}-\right)$ and $c=D_{m} D_{s}^{+} f\left(x_{2}-\right)$. Choose $x_{2}<\xi_{1}<\xi_{2}<x_{3}$ such that $\left(|b|+|c| m\left[x_{2}, \xi_{1}\right]\right) s_{x_{2}}\left(\xi_{1}\right)<\varepsilon$, and then, choose a continuous function $g$ on $\left(x_{1}, \xi_{2}\right)$ in such a way that $g=D_{m} D_{s}^{+} f$ on $\left(x_{1}, x_{2}\right), g=0$ on $\left[\xi_{1}, \xi_{2}\right)$, and that if we define $h(x)=b+\int_{\left[x_{2}, x\right]} g(y) m(d y)$, then $|h(x)| \leqq|b|+|c| m\left[x_{2}, \xi_{1}\right]$ on $\left[x_{2}, \xi_{1}\right]$ and $h\left(\xi_{1}\right)=0$. Define $\tilde{f}$ on $\left(x_{1}, \xi_{2}\right)$ by the right-hand side of (2.4) with $x_{0}=x_{2}$. By Lemma 2.3 and Remark 2.1 we see that $\tilde{f}$ is an extension of $[f]_{\left(x_{1}, x_{2}\right)}$ and belongs to $\mathfrak{D}\left(D_{m} D_{s}^{+} ;\left(x_{1}, \xi_{2}\right)\right)$. $f$ satisfies (2.6) and is flat on $\left[\xi_{1}, \xi_{2}\right)$. In a similar way we can get $\xi_{3}, \xi_{4}\left(\xi_{2}<\xi_{3}<\xi_{4}<x_{3}\right)$, and $\tilde{f}$ on $\left(\xi_{3}, x_{4}\right)$ which is flat on $\left(\xi_{3}, \xi_{4}\right]$. Finally, $\tilde{f}$ on $\left[\xi_{2}, \xi_{3}\right]$ is obtained by Lemma 2.4 .

REMARK 2.3. In the above proof, if $|b| \leqq k_{1}$ and $|c| \leqq k_{1}$, then $g$ can be chosen to satisfy $\|g\| \leqq k_{2}$, where $k_{2}$ is a constant which depends on $x_{2}, x_{3}, \varepsilon$, and $k_{1}$. For, choose $\xi_{1}$ such as $k_{1}\left(1+m\left[x_{2}, \xi_{1}\right]\right) s_{x_{2}}\left(\xi_{1}\right)<\varepsilon$, let $\xi_{0} \in\left(x_{2}, \xi_{1}\right)$ and let $g_{0}$ be a continuous function on $\left[\xi_{0}, \xi_{1}\right]$ vanishing at $\xi_{0}$ and $\xi_{1}$ and positive on $\left(\xi_{0}, \xi_{1}\right)$. Given $f$, we can choose $g$ as follows: $g(x)=c\left(\xi_{0}-x\right) /\left(\xi_{0}-x_{2}\right)$ for $x \in\left[x_{2}, \xi_{0}\right]$ and

$$
g(x)=-h\left(\xi_{0}\right)\left(\int_{\left(\xi_{0}, \xi_{1}\right]} g_{0}(y) m(d y)\right)^{-1} g_{0}(x) \text { for } x \in\left[\xi_{0}, \xi_{1}\right]
$$

This remark will be useful later.

LEMMA 2.6. Let $x_{1}<x_{2}<x_{3}<x_{4}$ and let $f(x)=c \varphi_{x_{0}}(x)$ on $\left(x_{1}, x_{2}\right)$ and $f(x)$ $=c^{\prime} \varphi_{x_{0}}(x)$ on $\left(x_{3}, x_{4}\right)$. Then, there is a function $\tilde{f} \in \mathcal{D}\left(D_{m} D_{s}^{+} ;\left(x_{1}, x_{4}\right)\right)$ such that $\tilde{f}=f$ on $\left(x_{1}, x_{2}\right) \cup\left(x_{3}, x_{4}\right)$ and

$$
\left(c \wedge c^{\prime}\right) \varphi_{x_{0}}(x) \leqq \tilde{f}(x) \leqq\left(c \vee c^{\prime}\right) \varphi_{x_{0}}(x)
$$

The same is true if we replace $\varphi_{x_{0}}$ by $\psi_{x_{0}}$ in the above statement. 
Proof. Assume $x_{0}<x_{2}<x_{3}$ or $x_{2}<x_{3}<x_{0}$. The other case is readily reduced to this case. Let $c<c^{\prime}$. Let $x_{2}<\xi_{1}<\xi_{2}<x_{3}$ and let $\tilde{f}=f$ on $\left(x_{1}, x_{2}\right) \cup\left(x_{3}, x_{4}\right)$, and

$$
\begin{array}{ll}
\tilde{f}(x)=c \varphi_{x_{0}}(x)+\int_{x_{2}}^{x} s(d y) \int_{\left(x_{2}, y\right]} g(z) m(d z) & \text { for } x \in\left[x_{2}, \xi_{1}\right), \\
\tilde{f}(x)=c^{\prime} \varphi_{x_{0}}(x)-\int_{x}^{x_{3}} s(d y) \int_{\left(y, x_{3}\right]} g(z) m(d z) & \text { for } x \in\left(\xi_{2}, x_{3}\right],
\end{array}
$$

where $g$ is positive continuous on $\left(x_{2}, \xi_{1}\right) \cup\left(\xi_{2}, x_{3}\right)$ and $g\left(x_{2}+\right)=g\left(x_{3}-\right)=0$. Then $\tilde{f} \in \mathfrak{D}\left(D_{m} D_{s}^{+} ;\left(x_{1}, \xi_{1}\right) \cup\left(\xi_{2}, x_{4}\right)\right)$ by Lemma 2.3. If we choose $\xi_{1}$ close to $x_{2}$ and $\xi_{2}$ close to $x_{3}$, we have $c \varphi_{x_{0}}(x)<\tilde{f}(x)<c^{\prime} \varphi_{x_{0}}(x)$ on $\left(x_{2}, \xi_{1}\right) \cup\left(\xi_{2}, x_{3}\right)$. In order to obtain $\tilde{f}$ on $\left(x_{1}, x_{4}\right)$, we have only to connect the two separate parts of $\tilde{f}$ obtained in this way first by a step function, and then using Lemmas 2.4 and 2.5 appropriately. The case $c=c^{\prime}$ is trivial and the case $c>c^{\prime}$ is treated similarly.

Using these lemmas, we can prove some assertions concerning how large the domain $\mathfrak{D}_{K}\left(D_{m} D_{s}^{+}\right)$is.

LEMMA 2.7. Let $f \in C_{K}(R)$ and $S(f) \subset\left[x_{1}, x_{2}\right]$. For any $\varepsilon>0$, we can find a function $f^{\prime} \in \mathscr{D}_{K}\left(D_{m} D_{s}^{+}\right)$such that $\left\|f-f^{\prime}\right\|<\varepsilon$ and $S\left(f^{\prime}\right) \subset\left[x_{1}, x_{2}\right]$.

Proof. First approximate $f$ by a step function vanishing near $x_{1}$ and $x_{2}$, and then use Lemma 2.4.

Lemma 2.8. Let $x_{0}$ be fixed. Let $f \in C_{K}(R)$ and $S(f) \subset\left[x_{1}, x_{2}\right]$. Given $\varepsilon>0$, we can find $f^{\prime} \in C_{K}(R)$ such that $f^{\prime} \cdot \varphi_{x_{0}} \in \mathfrak{D}_{K}\left(D_{m} D_{s}^{+}\right),\left\|f-f^{\prime}\right\|<\varepsilon$, and $S\left(f^{\prime}\right) \subset\left[x_{1}, x_{2}\right]$. The statement remains true if we replace $\varphi_{x_{0}}$ by $\psi_{x_{0}}$.

Proof. Suppose $x_{0} \notin\left(x_{1}, x_{2}\right)$. Approximate $f$ by a step function $g$ vanishing on $\left(-\infty, x_{1}+\delta\right) \cup\left(x_{2}-\delta,+\infty\right)$ for some $\delta>0$ and having an even number of jump points $\xi_{1}, \xi_{2}, \ldots, \xi_{n}$. Suppose $x_{1}<\xi_{1}<\cdots<\xi_{n}<x_{2}$ and $g(x)=c_{i}$ on $\left(\xi_{i}, \xi_{i+1}\right)$. Choose a function $h \in \mathfrak{D}_{K}\left(D_{m} D_{s}^{+}\right)$such that $h(x)=0$ on $\left(-\infty, \xi_{1}\right] \cup\left[\xi_{n},+\infty\right)$, $h(x)=c_{i} \varphi_{x_{0}}(x)$ on $\left(\xi_{i}, \xi_{i+1}\right)$ for even $i$, and

$$
\left(c_{i-1} \wedge c_{i+1}\right) \varphi_{x_{0}}(x) \leqq h(x) \leqq\left(c_{i-1} \vee c_{i+1}\right) \varphi_{x_{0}}(x)
$$

on $\left(\xi_{i}, \xi_{i+1}\right)$ for odd $i$, letting $c_{0}=c_{n}=0$. Such an $h$ exists by Lemma 2.6. Then $f^{\prime}(x)=h(x) / \varphi_{x_{0}}(x)$ is the desired function. In case $x_{0} \in\left(x_{1}, x_{2}\right)$, the proof is the same if only we choose $g$ and $f^{\prime}$ flat in a neighborhood of $x_{0}$.

Using Lemmas 2.4 and 2.5 combined with Remarks 2.2 and 2.3, we can prove another extension lemma.

LEMma 2.9. Given positive constants $k_{1}$ and $k_{2}\left(k_{1}>k_{2}\right)$, let $f \in \mathfrak{D}\left(D_{m} D_{s}^{+} ;\left(x_{1}, x_{2}\right)\right)$ have $\|f\|,\left\|D_{s}^{+} f\right\|$, and $\left\|D_{m} D_{s}^{+} f\right\|\left({ }^{11}\right)$ not exceeding $k_{1}$, let $D_{m} D_{s}^{+} f\left(x_{2}-\right)$ (hence also $f\left(x_{2}-\right)$ and $\left.D_{s}^{+} f\left(x_{2}-\right)\right)$ exist and let $f\left(x_{2}-\right) \geqq k_{2}$. Then we can find a constant $k_{3}$ such that there is an extension $f$ of $f$ to $\left(x_{1},+\infty\right)$ which is in $\mathfrak{D}\left(D_{m} D_{s}^{+} ;\left(x_{1},+\infty\right)\right)$,

$\left.{ }^{11}\right)$ We use the notation $\|g\|=\sup _{x}|g(x)|$ for any function $g$. 
positive on $\left[x_{2}, x_{3}\right)$ and zero on $\left[x_{3},+\infty\right)$ for some $x_{3}$, and satisfies $\|\tilde{f}\|,\left\|D_{m} D_{s}^{+} \tilde{f}\right\|$ $\leqq k_{3} . k_{3}$ is determined by $x_{2}, k_{1}$, and $k_{2}$. The similar assertion is true for extension to $\left(-\infty, x_{2}\right)$, too.

3. Lévy measures. The following theorem gives a sufficient condition for the existence of the Lévy measure and describes the order of its singularity and some other properties.

THEOREM 3.1. Let $\left\{T_{t}\right\}$ be an $M$-semigroup in $C_{0}(R)$ with infinitesimal generator (S) and assume that $\mathfrak{D}(\mathfrak{S}) \supset \mathfrak{D}_{K}\left(D_{m} D_{s}^{+}\right)$. Then,

(i) $\left\{T_{t}\right\}$ has the Lévy measure $\left\{n_{x}(d y) ; x \in R\right\}$.

(ii) $n_{x}$ is continuous with respect to $x$ in the sense that

$$
\lim _{x \rightarrow x_{0}} \int_{R \backslash\{x\}} f(y) n_{x}(d y)=\int_{R \backslash\left\{x_{0}\right\}} f(y) n_{x_{0}}(d y)
$$

for each $x_{0}$ and $f \in C_{K}(R)$ such that $S(f) \nexists x_{0}$.

(iii) $\lim _{|x| \rightarrow \infty} \int_{R \mid\{x\}} f(y) n_{x}(d y)=0$ for each $f \in C_{K}(R)$.

(iv) If $U$ is open, $V$ is bounded open, and $\bar{V} \subset U$, then $\sup _{x \in V} n_{x}(R \backslash U)<\infty$.

(v) (1.1) holds for all $x \in R$ and $f \in C_{0}(R)$ such that $S(f) \ngtr x$.

(vi) Let $E$ be compact. We have

$$
\begin{aligned}
& \int_{E \backslash\{x\}} \varphi_{x}(y) n_{x}(d y)<\infty \quad \text { if } x \notin \Gamma, \\
& \int_{E \backslash\{x\}}\left|s_{x}(y)\right| n_{x}(d y)<\infty \quad \text { if } x \in \Gamma .
\end{aligned}
$$

(vii) If $x_{0} \notin \Gamma$, then we have

$$
\lim _{t \rightarrow 0+} t^{-1} T_{t}\left(f \varphi_{x_{0}}\right)\left(x_{0}\right)=\int_{R \backslash\left\{x_{0}\right\}} f(y) \varphi_{x_{0}}(y) n_{x_{0}}(d y)
$$

for every $f \in C_{K}(R)$ such that $f\left(x_{0}\right)=0$. If $x_{0} \in \Gamma$, then

$$
\lim _{t \rightarrow 0+} t^{-1} T_{t}\left(f s_{x_{0}}\right)\left(x_{0}\right)=\int_{R \backslash\left\{x_{0}\right\}} f(y) s_{x_{0}}(y) n_{x_{0}}(d y)
$$

for every $f \in C_{K}(R)$ such that $f\left(x_{0}\right)=0$.

Proof. Let $x_{0} \in R$. For each $f \in \mathscr{D}_{K}\left(D_{m} D_{s}^{+}\right)$such that $f\left(x_{0}\right)=0, t^{-1} T_{t} f\left(x_{0}\right)$ tends to $\mathbb{S} f\left(x_{0}\right)$ as $t \rightarrow 0+$. Given $E=\left[x_{1}, x_{2}\right] \nexists x_{0}$, let $g$ be such that $g \in$ $\mathfrak{D}_{K}\left(D_{m} D_{s}^{+}\right), g \geqq 0,[g]_{E}=1$, and $g\left(x_{0}\right)=0$. Such $g$ is found by Lemma 2.4. For each $f \in C_{K}(R)$ such that $S(f) \subset E, t^{-1} T_{t} f\left(x_{0}\right)$ is convergent as $t \rightarrow 0+$. In fact, given $\varepsilon>0$, we can pick $f^{\prime} \in \mathfrak{D}_{K}\left(D_{m} D_{s}^{+}\right)$such that $\left\|f-f^{\prime}\right\|<\varepsilon$ and $S\left(f^{\prime}\right) \subset E$ (Lemma 2.7), and we have

$$
t^{-1} T_{t} f\left(x_{0}\right)=t^{-1} T_{t} f^{\prime}\left(x_{0}\right)+t^{-1} T_{t}\left(f-f^{\prime}\right)\left(x_{0}\right),
$$

the first term of which is convergent as $t \rightarrow 0+$ and the second term has absolute value $\leqq \varepsilon t^{-1} T_{t} g\left(x_{0}\right)$, which tends to $\varepsilon(S) g\left(x_{0}\right)$. Consequently, there exists a finite 
measure $n_{x_{0}}^{E}$ on $E$ such that $t^{-1} T_{t} f\left(x_{0}\right)$ tends to $\int_{E} f(y) n_{x_{0}}^{E}(d y) . E \subset E^{\prime}$ implies $n_{x_{0}}^{E}=n_{x_{0}}^{E^{\prime}}$ on $E$. Hence $n_{x_{0}}^{E}$ is extended to a measure $n_{x_{0}}$ on $R \backslash\left\{x_{0}\right\}$, which satisfies the conditions of Lévy measure. The assertion (ii) is proved through approximation of $f$ by functions of $\mathfrak{D}_{K}\left(D_{m} D_{s}^{+}\right)$. Note that the assertion is immediate if $f \in \mathfrak{D}_{K}\left(D_{m} D_{s}^{+}\right)$, since

$$
\int_{R \backslash\{x\}} f(y) n_{x}(d y)=\mathfrak{S} f(x), \quad x \notin S(f) .
$$

(iii) is also obvious for $f \in \mathfrak{D}_{K}\left(D_{m} D_{s}^{+}\right)$. For general $f \in C_{K}(R)$, use $f^{\prime} \in \mathscr{D}_{K}\left(D_{m} D_{s}^{+}\right)$ such that $f^{\prime} \geqq|f|$. In proving (iv), we may assume $U$ to be bounded. Let $g$ be such that $g \in \mathfrak{D}_{K}\left(D_{m} D_{s}^{+}\right), g \geqq 0$, and $[g]_{U}=1$ (Lemma 2.4). For each compact set $E \supset U$, choose $f$ such that $f \in \mathfrak{D}_{K}\left(D_{m} D_{s}^{+}\right), 0 \leqq f \leqq 1$, and $f=1$ on $E$ (Lemma 2.4). We have, for $x \in V$,

$$
\begin{aligned}
\mathscr{S} f(x) & =\int_{R \backslash U}(f-g)(y) n_{x}(d y)+\operatorname{Ss} g(x) \\
& \geqq n_{x}(E \backslash U)-\int_{R \backslash U} g(y) n_{x}(d y)+\operatorname{SS} g(x)
\end{aligned}
$$

by (3.5). It follows from $\mathfrak{S} f(x) \leqq 0$ that

$$
n_{x}(E \backslash U) \leqq \int_{R \backslash U} g(y) n_{x}(d y)-\operatorname{Bsg} g(x)
$$

The right-hand side is independent of $E$ and bounded on $V$, because the first term is $\leqq \circlearrowleft s g^{\prime}(x)$ if we choose $g^{\prime} \in \mathscr{D}_{K}\left(D_{m} D_{s}^{+}\right)$satisfying $g^{\prime} \geqq 0, g^{\prime}=0$ on $V$, and $g^{\prime} \geqq g$ on $R \backslash U$ (Lemma 2.4). Hence we obtain (iv). Let $P_{t, x}(d y)$ be the measure such that $T_{t} f(x)=\int f(y) P_{t, x}(d y)$. By the same idea we get finiteness of $\sup _{t} t^{-1} P_{t, x}(R \backslash U)$ for $x \in U$, and hence, (v) is proved through approximation by functions of $C_{K}(R)$.

Proceeding to the proof of (vi) and (vii), let $x \in R$ be fixed, and let

$$
t^{-1} \varphi_{x}(y) P_{t, x}(d y)=Q_{t, x}(d y) .
$$

If $f \in C_{K}(R)$ satisfies $f \cdot \varphi_{x} \in \mathscr{D}_{K}\left(D_{m} D_{s}^{+}\right)$, then $\int f(y) Q_{t, x}(d y)$ tends to $\mathscr{B S}\left(f \varphi_{x}\right)(x)$ as $t \rightarrow 0+$. We claim that $\int f(y) Q_{t, x}(d y)$ converges for all $f \in C_{K}(R)$. Let $S(f)$ $\subset\left[x_{1}, x_{2}\right]$. Choose $g \in C_{K}(R)$ such that $g \cdot \varphi_{x} \in \mathfrak{D}_{K}\left(D_{m} D_{s}^{+}\right), g \geqq 0$, and $g=1$ on $\left[x_{1}, x_{2}\right]$, using Lemma 2.6. Further, we can choose, for any $\varepsilon>0$, a function $f^{\prime} \in C_{K}(R)$ such that $f^{\prime} \cdot \varphi_{x} \in \mathfrak{D}_{K}\left(D_{m} D_{s}^{+}\right),\left\|f-f^{\prime}\right\|<\varepsilon$, and $S\left(f^{\prime}\right) \subset\left[x_{1}, x_{2}\right]$ by using Lemma 2.8. Then, $\int f^{\prime} Q_{t, x}(d y)$ converges and $\int\left(f-f^{\prime}\right) Q_{t, x}(d y)$ has absolute value $\leqq \varepsilon \int g Q_{t, x}(d y)$. Hence, $\int f Q_{t, x}(d y)$ converges as $t \rightarrow 0+$. There is a measure $R_{x}(d y)$ on $R$ finite for compact sets such that the limit is represented by $\int f R_{x}(d y)$. If $f$ vanishes in a neighborhood of $x$, then the limit also equals $\int f \varphi_{x} n_{x}(d y)$. Hence, $R_{x}(d y)=\varphi_{x}(y) n_{x}(d y)$ on $R \backslash\{x\}$. This proves (3.1) for all $x$ and (3.3) for all $x_{0}$. The same fact can be proved for $\psi_{x}$ instead of $\varphi_{x}$, and we get (3.2) and (3.4), since $\psi_{x}(y)-\varphi_{x}(y)=m\{x\} s_{x}(y)$. The proof of Theorem 3.1 is complete. 
Corollary. Let $f \in \mathfrak{D}_{K}\left(D_{m} D_{s}^{+}\right)$and let $U$ be a bounded open interval. The integral

$$
\int_{R \backslash\{x\}}\left[f(y)-f(x)-\chi_{U}(y) s_{x}(y) D_{s}^{+} f(x)\right] n_{x}(d y)
$$

exists for $x \in U$ and is measurable $\left({ }^{12}\right)$ with respect to $x$. The same is true with $D_{s}^{+} f$ replaced by $D_{s}^{-} f$.

Proof. Existence of the integral is a consequence of (iv), (vi), and Lemma 2.3. If $E$ and $E^{\prime}$ are disjoint compact sets and if $g(x, y)$ is a bounded jointly measurable function on $E \times E^{\prime}$, then $\int_{E^{\prime}} g(x, y) n_{x}(d y)$ is measurable on $E$ by a standard argument. Let $g(x, y)$ be the integrand in (3.6), $E=\left[x_{0}, x_{0}+1 / n\right]$, and $E^{\prime}=\left[x_{0}+2 / n\right.$, $\left.x_{0}+n\right]$. Then we see that $\int_{[x+2 / n, x+n-1 / n]} g(x, y) n_{x}(d y)$ is measurable on $E$, hence on $R$. Therefore $\int_{(x,+\infty)} g(x, y) n_{x}(d y)$ is measurable on $U$, and so is the integral on $(-\infty, x)$.

THEOREM 3.2. Under the same assumption as in Theorem 3.1, suppose, further, $\mathfrak{D}\left(S_{S}\right) \supset \mathfrak{D}_{0}\left(D_{m} D_{s}^{+}\right)$. Then,

$$
\sup _{x \in E} \int_{E \backslash\{x\}}\left(\varphi_{x}(y)+\psi_{x}(y)\right) n_{x}(d y)<\infty
$$

for any compact set $E$.

Proof. Let $A=D_{m} D_{s}^{+}$with domain $\mathscr{D}(A)=\mathscr{D}_{0}\left(D_{m} D_{s}^{+}\right)$. We claim $A$ is a closed operator in $C_{0}(R)$. Suppose $f_{n} \in \mathfrak{D}(A), A f_{n}=g_{n},\left\|f-f_{n}\right\| \rightarrow 0$, and $\left\|g-g_{n}\right\| \rightarrow 0$. We will prove $f \in \mathfrak{D}(A)$ and $A f=g$. Let $x_{1}<x_{2}$ and $x_{1}, x_{2} \notin \Gamma$. As Feller [3] shows, there are the Green function $G(x, y)$ and the minimal harmonic functions $H_{1}(x)$ and $H_{2}(x)$ relative to $D_{m} D_{s}^{+}$on $\left[x_{1}, x_{2}\right]$, and $f_{n}$ is represented as

$$
f_{n}(x)=\int_{x_{1}}^{x_{2}} G(x, y) g_{n}(y) m(d y)+H_{1}(x) f_{n}\left(x_{1}\right)+H_{2}(x) f_{n}\left(x_{2}\right)
$$

It follows that the same equality holds with $f$ and $g$ replacing $f_{n}$ and $g_{n}$, which implies $f \in \mathfrak{D}(A)$ and $A f=g$. Hence, $A$ is closed. (S) is also closed since it is an infinitesimal generator. From this closedness of two operators, we get an estimate

$$
\|\mathfrak{s} f\| \leqq k_{1}\left\|D_{m} D_{s}^{+} f\right\|+k_{2}\|f\|, \quad f \in \mathfrak{D}_{0}\left(D_{m} D_{s}^{+}\right),
$$

by application of the closed graph theorem (see Yosida [16, Chapter II, §6]). Let $E$ be a compact set and $U$ be a bounded open interval, $U \supset E$. Applying Lemma 2.9 to $f=\left[\varphi_{x}\right]_{U}$ with $x \in E$, we find extensions $\tilde{\varphi}_{x}$ of $\left[\varphi_{x}\right]_{U}$ such that $\tilde{\varphi}_{x} \in \mathscr{D}_{K}\left(D_{m} D_{s}^{+}\right)$,

( $\left.{ }^{12}\right)$ We mean Borel measurable by measurable. 
$\tilde{\varphi}_{x} \geqq 0,\left\|\tilde{\varphi}_{x}\right\| \leqq k_{3}$, and $\left\|D_{m} D_{s}^{+} \tilde{\varphi}_{x}\right\| \leqq k_{3} . k_{3}$ is a constant independent of $x \in E$. Let $g_{x}(y)=\tilde{\varphi}_{x}(y) / \varphi_{x}(y)$. We have

$$
\begin{aligned}
\int_{E \backslash\{x\}} \varphi_{x}(y) n_{x}(d y) & \leqq \int_{R \backslash\{x\}} g_{x}(y) \varphi_{x}(y) n_{x}(d y) \leqq \int_{R} g_{x}(y) R_{x}(d y) \\
& =\lim _{t \rightarrow 0+} \int_{R} g_{x}(y) Q_{t, x}(d y)=\operatorname{BS}\left(\tilde{\varphi}_{x}\right)(x) \leqq\left(k_{1}+k_{2}\right) k_{3}
\end{aligned}
$$

by (3.8). A similar argument can be made for $\psi_{x}$ and the proof is complete.

\section{Representation of infinitesimal generators.}

THEOREM 4.1. Suppose that $\left\{T_{t}\right\}$ is an M-semigroup in $C_{0}(R)$ with infinitesimal generator (S) such that $\mathfrak{D}(\mathbb{S S}) \supset \mathfrak{D}_{K}\left(D_{m} D_{s}^{+}\right)$. Then,

(i) For each bounded open interval $U$, there are unique functions $a(x), b(x)$, and $c(x)$ on $U \backslash \Gamma$ such that for every $f \in \mathfrak{D}_{K}\left(D_{m} D_{s}^{+}\right)$and $x \in U \backslash \Gamma \mathfrak{S} f(x)$ is represented by (1.4), where $n_{x}(d y)$ is the Lévy measure for $\left\{T_{t}\right\} . a, b$, and c are measurable, a and $c$ are independent of $U$ and satisfy (1.5).

(ii) For each $x \in \Gamma$ there are unique $a^{+}(x), a^{-}(x)$, and $c(x)$ such that for every $f \in \mathfrak{D}_{K}\left(D_{m} D_{s}^{+}\right)$Sf $f(x)$ is represented by (1.6). They satisfy (1.7).

RemarK 4.1. Actually, $a$ and $b$ are also defined on the whole $R$ and $U$, respectively, and we have

$$
\begin{aligned}
\mathfrak{S} f(x)= & a(x) D_{m} D_{s}^{+} f(x)+b(x) D_{s}^{+} f(x)+c(x) f(x) \\
& +\int_{R \backslash\{x\}}\left[f(y)-f(x)+\chi_{U}(y) s_{x}(y) D_{s}^{+} f(x)\right] n_{x}(d y)
\end{aligned}
$$

for all $x \in U$. (Note that $D_{s}^{+} f=D_{s}^{-} f=D_{s} f$ outside of $\Gamma$.) $c$ is lower semicontinuous on $R, a$ is upper semicontinuous at points $\notin \Gamma$, and $b$ is continuous at $x$ such that $n_{x}\left(\left\{x_{1}\right\}\right)=n_{x}\left(\left\{x_{2}\right\}\right)=0$ where $x_{1}$ and $x_{2}$ are the boundary points of $U$. Using any function $\rho \in C_{K}(R)$ which is 1 on $U$, we can also have

$$
\begin{aligned}
\text { SS } f(x)= & a(x) D_{m} D_{s}^{+} f(x)+b^{\prime}(x) D_{s}^{+} f(x)+c^{\prime}(x) f(x) \\
& +\int_{R \backslash\{x\}}\left[f(y)-\rho(y)\left(f(x)+s_{x}(y) D_{s}^{+} f(x)\right)\right] n_{x}(d y)
\end{aligned}
$$

for all $x \in U$. This time, $b^{\prime}$ and $c^{\prime}$ are continuous. A similar remark can be made with $D_{s}^{+} f(x)$ replaced by $D_{s}^{-} f(x)$.

Proof. Let $U=\left(x_{1}, x_{2}\right), x \in U$, and let $\tilde{s}_{x}, \tilde{\varphi}_{x}$, and $\tilde{\rho}$ be functions in $\mathfrak{D}_{K}\left(D_{m} D_{s}^{+}\right)$ which coincide on $U$ with $s_{x}, \varphi_{x}$, and 1 , respectively, and $\tilde{\varphi}_{x} \geqq 0,1 \geqq \tilde{\rho} \geqq 0$. Given $f \in \mathbb{F}_{K}\left(D_{m} D_{s}^{+}\right)$define $g_{x}(y)$ by

$$
f(y)=\tilde{\rho}(y) f(x)+\tilde{s}_{x}(y) D_{s}^{+} f(x)+\tilde{\varphi}_{x}(y) D_{m} D_{s}^{+} f(x)+g_{x}(y) .
$$


Since $g_{x} \in C_{K}(R)$ and $g_{x}(y) / \varphi_{x}(y)$ tends to 0 as $y \rightarrow x$ (use Lemma 2.3), we have (s) $f(x)=\operatorname{ss} \tilde{\rho}(x) \cdot f(x)+\operatorname{sss}\left(\tilde{s}_{x}\right)(x) \cdot D_{s}^{+} f(x)+\operatorname{sis}\left(\tilde{\varphi}_{x}\right)(x) \cdot D_{m} D_{s}^{+} f(x)+\int_{R \backslash\{x\}} g_{x}(y) n_{x}(d y)$ by Theorem 3.1 (vii). We obtain (4.1) from this because

$$
\begin{aligned}
\int g_{x}(y) n_{x}(d y)= & \int\left[f(y)-f(x)-\chi_{U}(y) s_{x}(y) D_{s}^{+} f(x)\right] n_{x}(d y) \\
& +f(x) \int[1-\tilde{\rho}(y)] n_{x}(d y)+D_{s}^{+} f(x) \int\left[\chi_{U}(y) s_{x}(y)-\tilde{s}_{x}(y)\right] n_{x}(d y) \\
& -D_{m} D_{s}^{+} f(x) \int \tilde{\varphi}_{x}(y) n_{x}(d y) .
\end{aligned}
$$

It follows from

$$
\text { (S) } \tilde{\rho}(x)=c(x)+\int_{R \backslash\{x\}}[\tilde{\rho}(y)-1] n_{x}(d y)
$$

that $c$ is unique and measurable. This formula also implies that $c$ is independent of the choice of $U$. In fact, if $U^{\prime}$ is another bounded open interval containing $x$ and if $\tilde{\rho}^{\prime}$ is a function in $\mathfrak{D}_{K}\left(D_{m} D_{s}^{+}\right)$equal to 1 on $U^{\prime}$, then the above formula is valid with $\tilde{\rho}^{\prime}$ replacing $\tilde{\rho}$. We see that $c(x) \leqq 0$ by choosing $U$ large and noting $(s) \rho(x) \leqq 0$, and, at the same time, $c$ turns out to be lower semicontinuous, being the increasing limit of continuous functions. If $\tilde{s} \in \mathfrak{D}_{K}\left(D_{m} D_{s}^{+}\right)$is an extension of $[s]_{U}$, we have

$$
\text { SS } \tilde{s}(x)=b(x)+c(x) s(x)+\int\left[\tilde{s}(y)-s(x)-\chi_{U}(y) s_{x}(y)\right] n_{x}(d y),
$$

which implies uniqueness and measurability of $b$. Let us write (4.1) into (4.2). Then, $c^{\prime}$ and $b^{\prime}$ are continuous on $U$, since

$$
\begin{aligned}
& \text { (SS) } \tilde{\rho}(x)=c^{\prime}(x)+\int_{R \backslash(x)}[\tilde{\rho}(y)-\rho(y)] n_{x}(d y), \\
& \text { SS } \tilde{s}(x)=b^{\prime}(x)+c^{\prime}(x) s(x)+\int_{R \backslash\{x\}}[\tilde{s}(y)-\rho(y) s(y)] n_{x}(d y) .
\end{aligned}
$$

Since $B\left(\tilde{\varphi}_{x}\right)(x)=a(x)+\int_{R \backslash\{x\}} \tilde{\varphi}_{x}(y) n_{x}(d y), a$ is unique and is independent of the choice of $U$. We have $a(x) \geqq 0$ for fixed $x$ by choosing $U$ and $S\left(\tilde{\varphi}_{x}\right)$ very small and noting $\mathscr{G}\left(\tilde{\varphi}_{x}\right)(x) \geqq 0$. Fix a point $x_{0}<x_{1}$ and let $\tilde{\varphi}=\tilde{\varphi}_{x_{0}}$. By the equality

$$
\begin{aligned}
& \text { SS } \tilde{\varphi}(x)=a(x)+b^{\prime}(x) m\left(x_{0}, x\right]+c^{\prime}(x) \tilde{\varphi}(x) \\
& +\int_{R \backslash\{x\}}\left[\tilde{\varphi}(y)-\rho(y)\left(\tilde{\varphi}(x)+s_{x}(y) m\left(x_{0}, x\right]\right)\right] n_{x}(d y),
\end{aligned}
$$

$a$ is measurable on $U$ and upper semicontinuous at points $\notin \Gamma$. In fact, let $\xi \notin \Gamma$ and let $\left\{\sigma_{n}\right\}$ be an increasing sequence of continuous functions such that $1 \geqq \sigma_{n} \geqq 0$, $\sigma_{n}=1$ outside of $(\xi-2 / n, \xi+2 / n)$ and $\sigma_{n}=0$ on $(\xi-1 / n, \xi+1 / n)$. Let $g(x)$ be the integral in (4.3) and $g_{n}(x)$ be the same integral with the integrand multiplied by $\sigma_{n}(y)$. Then, $g_{n}$ is continuous at $\xi, g_{n} \leqq g$, and $g_{n}(\xi)$ increases to $g(\xi)$ as $n \rightarrow \infty$. 
Hence, $g$ is lower semicontinuous at $\xi$, and the upper semicontinuity of $a$ at $\xi$ follows. The continuity of $b$ at $x$ such that $n_{x}\left(\left\{x_{1}\right\}\right)=n_{x}\left(\left\{x_{2}\right\}\right)=0$ is proved from

$$
\left(\xi \tilde{s} \tilde{s}(x)=b(x)+c^{\prime}(x) s(x)+\int_{R \backslash U}[\tilde{s}(y)-\rho(y) s(x)] n_{x}(d y) .\right.
$$

The proof of (ii) is as follows. Let $x \in \Gamma$. Noting that

$$
D_{m} D_{s}^{+} f(x)=(m\{x\})^{-1}\left(D_{s}^{+} f(x)-D_{s}^{-} f(x)\right)
$$

and using (3.2), we can rewrite (4.1) into (1.6), and we have $a^{-}(x)=a(x) \geqq 0$ and $c(x) \leqq 0$. The uniqueness of $a^{+}, a^{-}, c$ in (1.6) follows from that of $a, b, c$ in (4.1), since (1.6) can be written into (4.1) conversely. Let $\tilde{\psi}_{x}$ be a nonnegative function in $\mathfrak{D}_{K}\left(D_{m} D_{s}^{+}\right)$which equals $\psi_{x}$ in a neighborhood of $x$. Then,

$$
0 \leqq \mathfrak{S}\left(\tilde{\psi}_{x}\right)(x)=a^{+}(x)+\int \tilde{\psi}_{x}(y) n_{x}(d y)
$$

and hence we get $a^{+}(x) \geqq 0$, letting $S\left(\tilde{\psi}_{x}\right)$ be small enough. The proof of Theorem 4.1 and Remark 4.1 is complete.

REMARK 4.2. In Theorem 4.1, $\lim _{t \rightarrow 0+} t^{-1}\left(T_{t} f(x)-f(x)\right)$ exists for each point $x \in R$ if $f \in \mathscr{D}\left(D_{m} D_{s}^{+} ; R\right) \cap C_{0}(R)$. The limit is represented in the same way. In fact, such $f$ is the sum of a function in $\mathfrak{D}_{K}\left(D_{m} D_{s}^{+}\right)$and a function in $C_{0}(R)$ which vanishes in a neighborhood of $x$ (Lemma 2.5). Theorem 3.1 (v) applies to the latter.

Adding some observations to the above proof as we did in the proof of Theorem 3.2 , we get the following result.

THEOREM 4.2. Suppose, further, $\mathfrak{D}(\circlearrowleft) \supset \mathfrak{D}_{0}\left(D_{m} D_{s}^{+}\right)$in Theorem 4.1. Then, $a, a^{+}$, $a^{-}$, and $c$ are bounded on any compact set in $R$ and $b$ is bounded on any compact set in $U$.

5. Generation of $M$-semigroups. In this section we refer to the following restrictions on $m$ and $s$.

Property I. $D_{m} D_{s}^{+}$with domain $\mathscr{D}_{0}\left(D_{m} D_{s}^{+}\right)$generates an $M$-semigroup in $C_{0}(R)$.

PROPERTY II. There is a positive constant $\alpha$ such that for each $x \in R \backslash \Gamma$ there exist $y_{x}$ and $y_{x}^{\prime}$ which satisfy $y_{x} \leqq x \leqq y_{x}^{\prime},\left(s\left(y_{x}^{\prime}\right)-s\left(y_{x}\right)\right)^{-1}\left(\varphi_{x}\left(y_{x}\right)+\varphi_{x}\left(y_{x}^{\prime}\right)\right) \leqq \alpha$, and $\sup _{x \in R \mid \Gamma}\left(s\left(y_{x}^{\prime}\right)-s\left(y_{x}\right)\right)^{-1}<\infty$.

PROPERTY III. The constant $\alpha$ in Property II can be chosen arbitrarily small.

Property IV. $m$ is continuous, that is, $\Gamma$ is void.

Lemma 5.1. Suppose that $m$ and $s$ have Property II. Then, there is a constant $\beta$ such that

$$
\left\|D_{s}^{+} f\right\|=\left\|D_{s}^{-} f\right\| \leqq \alpha\left\|D_{m} D_{s}^{+} f\right\|+\beta\|f\|
$$

for all $f \in \mathscr{D}_{0}\left(D_{m} D_{s}^{+}\right)$. 
Proof. The first equality is clear from $D_{s}^{-} f(x)=D_{s}^{+} f(x-)$. We have, by Lemma 2.3,

$$
f\left(y^{\prime}\right)-f(y)=s_{y}\left(y^{\prime}\right) D_{s}^{+} f(x)+\int_{x}^{y^{\prime}} s(d z) \int_{(x, z]} g(w) m(d w)-\int_{y}^{x} s(d z) \int_{(z, x]} g(w) m(d w)
$$

where $g=D_{m} D_{s}^{+} f$ and $y \leqq x \leqq y^{\prime}$. Hence

$$
\left|D_{s}^{+} f(x)\right| \leqq s_{y}\left(y^{\prime}\right)^{-1}\left|f\left(y^{\prime}\right)-f(y)\right|+s_{y}\left(y^{\prime}\right)^{-1}\left(\varphi_{x}(y)+\varphi_{x}\left(y^{\prime}\right)\right) \sup _{z \in\left(y, y^{\prime}\right)}|g(z)|
$$

and we get (5.1) by Property II. Note that $R \backslash \Gamma$ is dense.

The next lemma is a special case of a result [4] in general Banach lattices (see [11] in case $k_{1}<1 / 2$ ).

Lemma 5.2. Suppose that $A$ generates an $M$-semigroup in $C_{0}(R)$ and $B$ is a linear operator with domain $\mathfrak{D}(B) \supset \mathfrak{D}(A)$. Let $\mathbb{S}=A+B$. If $\mathbb{S S}$ is dispersive and if there are constants $k_{1}<1$ and $k_{2}<+\infty$ such that

$$
\|B f\| \leqq k_{1}\|A f\|+k_{2}\|f\| \quad \text { for } f \in \mathfrak{D}(A),
$$

then (S) generates an $M$-semigroup.

We will give three theorems.

THEOREM 5.1. Suppose that $m$ and $s$ have Properties I and II. Let (S) be an operator in $C_{0}(R)$ with domain $\mathfrak{D}(\mathbb{S})=\mathfrak{D}_{0}\left(D_{m} D_{s}^{+}\right)$such that, for each $x \in R$, there are $a$ measure $n_{x}(d y)$ on $R \mid\{x\}$ and an open interval $U_{x}$ containing $x$ and the following two conditions are satisfied:

(i) There are functions $b$ and $c$ on $R \backslash \Gamma$ such that (1.4) holds on $R \backslash \Gamma$ for $f \in \mathfrak{D}(\mathbb{B S})$ with $a(x)$ replaced by 1 and $U$ replaced by $U_{x}$, and they satisfy

$$
\begin{array}{r}
\alpha\|b\|+\sup _{x \in R \mid \Gamma} \int_{U_{x}} \varphi_{x}(y) n_{x}(d y)<1, \\
\quad \sup _{x \in R \mid \Gamma} n_{x}\left(R \backslash U_{x}\right)<\infty, \\
\|b\|<\infty, \quad\|c\|<\infty, \text { and } c \leqq 0 .
\end{array}
$$

(ii) There are functions $a^{+}, a^{-}$, and $c$ on $\Gamma$ such that (1.6) holds on $\Gamma$ for $f \in$ $\mathfrak{D}(\mathbb{B})$ and they satisfy (1.7),

$$
\begin{gathered}
\sup _{x \in \Gamma}(m\{x\})^{-1}\left(\left|a^{+}(x)-1\right|+\left|a^{-}(x)-1\right|\right)+\sup _{x \in \Gamma} \int_{U_{x}}\left|s_{x}(y)\right| n_{x}(d y)<\alpha^{-1}, \\
\sup _{x \in \Gamma} n_{x}\left(R \backslash U_{x}\right)<\infty \\
\|c\|<\infty
\end{gathered}
$$

Then (S) generates an $M$-semigroup in $C_{0}(R)$. 
Proof. The conditions in Lemma 5.2 are checked for $A=D_{m} D_{s}^{+}$and $B=(B)-A$. In fact, since $A$ generates an $M$-semigroup (Property $\mathrm{I}$ ) and $B S$ is dispersive, it is enough to establish the estimation in Lemma 5.2. Let $x \notin \Gamma$. We have

$$
|B f(x)| \leqq\|b\|\left\|D_{s}^{+} f\right\|+\|c\|\|f\|+\int_{U_{x}} \varphi_{x}(y) n_{x}(d y)\left\|D_{m} D_{s}^{+} f\right\|+2 n_{x}\left(R \backslash U_{x}\right)\|f\|
$$

and, by using Property II and Lemma 5.1, we get $|B f(x)| \leqq \alpha^{\prime}\|A f\|+\beta^{\prime}\|f\|$ where $\alpha^{\prime}$ is the left-hand side of (5.3) and $\beta^{\prime}$ is some constant. If $x \in \Gamma$, then we have

$$
\begin{aligned}
B f(x)= & (m\{x\})^{-1}\left[\left(a^{+}(x)-1\right) D_{s}^{+} f(x)-\left(a^{-}(x)-1\right) D_{s}^{-} f(x)\right]+c(x) f(x) \\
& +\int_{R \backslash\{x\}}[f(y)-f(x)] n_{x}(d y),
\end{aligned}
$$

and hence, $|B f(x)| \leqq \alpha^{\prime \prime}\|A f\|+\beta^{\prime \prime}\|f\|$ where $\alpha^{\prime \prime}$ is $\alpha$ times the left-hand side of (5.6) and $\beta^{\prime \prime}$ is some constant. Since $\alpha^{\prime}$ and $\alpha^{\prime \prime}$ are both less than 1, the proof is complete.

TheORem 5.2. Assume Properties I, III, and IV for $m$ and $s$. Let (5) be the same as in Theorem 5.1 except that condition (ii) is dropped and that $\Gamma=\varnothing$ and $\alpha=0$ in condition (i). Then, $b_{5}$ generates an M-semigroup in $C_{0}(R)$.

Proof. This is easily reduced to Theorem 5.1.

THEOREM 5.3. Assume Property I for $m$ and s. Let (Is) be a dispersive operator in $C_{0}(R)$ with domain $\mathfrak{D}(\xi)=\mathfrak{D}_{0}\left(D_{m} D_{s}^{+}\right)$. Suppose there are a measure $n_{x}(d y)$ on $R \backslash\{x\}$ for each $x$, an open interval $U_{x}$ containing $x$ for each $x$, a bounded function $c(x)$, and a subset $\Gamma^{\prime}$ of $\Gamma$ such that we have

(5.9) \&f $f(x)=D_{m} D_{s}^{+} f(x)+c(x) f(x)+\int_{R \backslash\{x\}}\left[f(y)-f(x)-\chi_{U_{x}}(y) s_{x}(y) D_{s}^{ \pm} f(x)\right] n_{x}(d y)$ for $f \in \mathfrak{D}(\mathbb{S})$, where $D_{s}^{ \pm} f(x)$ stands for $D_{s}^{+} f(x)$ or $D_{s}^{-} f(x)$ according as $x \in R \backslash \Gamma^{\prime}$ or $x \in \Gamma^{\prime}$, and such that

$$
\sup _{x \in R \mid \Gamma^{\prime}} \int_{U_{x}} \varphi_{x}(y) n_{x}(d y)<1, \sup _{x \in \Gamma^{\prime}} \int_{U_{x}} \psi_{x}(y) n_{x}(d y)<1,
$$

and

$$
\sup _{x \in R} n_{x}\left(R \backslash U_{x}\right)<\infty .
$$

Then, (S) generates an $M$-semigroup in $C_{0}(R)$.

Proof. Letting $A=D_{m} D_{s}^{+}$and $B=(\xi)-A$, one can readily check the conditions in Lemma 5.2.

REMARK 5.1. Instead of dispersiveness of (\$5) it suffices to assume $c \leqq 0$ on $R$ and

$$
(m\{x\})^{-1} \mp \int_{U_{x}} s_{x}(y) n_{x}(d y) \geqq 0
$$

on $\Gamma$. Here $\mp$ stands for - or + according as $x \in \Gamma \backslash \Gamma^{\prime}$ or $x \in \Gamma^{\prime}$. 
We make some remarks on Properties I-IV.

REMARK 5.2. If we use Feller's classification of the boundary points $-\infty$ and $+\infty$ into four sorts: regular, exit, entrance, and natural ([3], [7], or [16]), Property I is equivalent to that neither $-\infty$ nor $+\infty$ is an entrance boundary. For the proof, see Itô [7, §62].

Remark 5.3. If the measure $m$ is finite, then $m$ and $s$ have Property II with $\alpha=$ the total measure of $m$. This is because

$$
\left(s\left(y^{\prime}\right)-s(y)\right)^{-1}\left(\varphi_{x}\left(y^{\prime}\right)+\varphi_{x}(y)\right) \leqq m\left(y, y^{\prime}\right] \text { for } y \leqq x \leqq y^{\prime}, y \neq y^{\prime} .
$$

Remark 5.4. The following fact is useful in checking Property II: Let $K$ be compact and let $\alpha_{0}$ be the supremum of $m\{x\}$ for $x \in K \cap \Gamma$. Given any $\varepsilon>0$, we can find $y_{x}$ and $y_{x}^{\prime}$ for each $x \in K$ such that $y_{x} \leqq x \leqq y_{x}^{\prime}$,

$$
\left(s\left(y_{x}^{\prime}\right)-s\left(y_{x}\right)\right)^{-1}\left(\varphi_{x}\left(y_{x}\right)+\varphi_{x}\left(y_{x}^{\prime}\right)\right)<\alpha_{0}+\varepsilon
$$

and $\sup _{x \in K}\left(s\left(y_{x}^{\prime}\right)-s\left(y_{x}\right)\right)^{-1}<\infty$. In fact, we can choose $y_{x}=x$ and $y_{x}^{\prime}=x+\delta, \delta$ being independent of $x$.

The following two remarks are consequences of the preceding remark and (5.12).

REMARK 5.5. If $m$ is finite and continuous, then it has Property III for every $s$.

REMARK 5.6. Let $s(x)=x$ and assume $\lim \sup _{x \rightarrow-\infty} m(x-\delta, x]=\alpha^{-}<\infty$ and $\lim \sup _{x \rightarrow+\infty} m[x, x+\delta)=\alpha^{+}<\infty$ for some $\delta>0$. Then, they have Property II for any $\alpha>\alpha^{+} \vee \alpha^{-} \vee \sup _{x \in \Gamma} m\{x\}$. Such is the case if $s(x)=x$ and $m=$ const $x$ Lebesgue measure.

EXAMPLE 5.1. Let $s(x)=x$ and let $n_{x}(d y)$ be const $\times|y-x|^{\lambda-1} d y, \lambda>-1$, for $0<|y-x|<1$ and 0 for $|y-x| \geqq 1$. Assume Properties I and II, and assume, further, that we can choose

$$
y_{x}=x \text { near }+\infty \text { and } y_{x}^{\prime}=x \text { near }-\infty
$$

in Property II. It follows from (5.2) that $D_{s}^{+} f(x)$ tends to 0 as $|x| \rightarrow \infty$ for $f \in$ $\mathfrak{D}_{0}\left(D_{m} D_{s}^{+}\right)$. Let

$$
\operatorname{sif} f(x)=D_{m} D_{s}^{+} f(x)+\int_{0<|y-x|<1}[f(y)-f(x)] n_{x}(d y)
$$

for $f \in \mathfrak{I}_{0}\left(D_{m} D_{s}^{+}\right)$. Then, (is carries $\mathfrak{I}_{0}\left(D_{m} D_{s}^{+}\right)$into $C_{0}(R)$. If moreover,

$$
\sup _{x \in R \mid \Gamma^{\prime}} \int_{0<|y-x|<1} \varphi_{x}(y) n_{x}(d y)<1 \quad \text { and } \sup _{x \in \Gamma^{\prime}} \int_{0<|y-x|<1} \psi_{x}(y) n_{x}(d y)<1
$$

for some $\Gamma^{\prime} \subset \Gamma$, then $(3)$ generates an $M$-semigroup in $C_{0}(R)$ by virtue of Theorem 5.3.

The measure $n_{x}(d y)$ above has a mild singularity: it satisfies (3.2) for all $x$. Let us consider a more singular case: $\lambda=-1$. As the next example shows, the condition in Theorem 5.1 or 5.3 that $(5)$ maps $\mathfrak{D}_{0}\left(D_{m} D_{s}^{+}\right)$into $C_{0}(R)$ is a fairly strong restriction in case $m$ is not continuous. In case $m$ is continuous, we will give in Example 5.3 a sufficient condition for application of Theorem 5.2. 
EXAMPLE 5.2. Suppose $s(x)=x$ and $m=$ the Lebesgue measure added to a unit mass at the origin, and let

$$
B f(x)=\int_{0<|y-x|<1}\left[f(y)-f(x)-(y-x) D_{s} f(x)\right]|y-x|^{-2} d y
$$

for $f \in \mathfrak{D}_{0}\left(D_{m} D_{s}^{+}\right)$and $x \neq 0$. Then, $B$ cannot carry $\mathfrak{I}_{0}\left(D_{m} D_{s}^{+}\right)$into $C_{0}(R)$, no matter how $B f(0)$ is defined. In fact, if $f$ is such that $D_{m} D_{s}^{+} f=1$ near the origin, then $B f(0+)=+\infty$.

Example 5.3. Assume Properties I, III, and IV for $m$ and $s$. For every $x$, let $n_{x}(d y)=\pi^{-1}|y-x|^{-2} d y$ on $R \backslash\{x\}$. Assume that

$$
\sup _{x \in R} \int_{0<|y-x|<n} \varphi_{x}(y) n_{x}(d y)<1
$$

for some $h>0$ and that $\int_{0<|y-x|<\delta} \varphi_{x}(y) n_{x}(d y)$ tends to 0 uniformly on any compact set as $\delta \rightarrow 0+$. Let $b$ and $c$ be bounded continuous, $c \leqq 0$, and $\lim _{|x| \rightarrow \infty} b(x)$ $=0$, and let $(S f(x)$ be defined by (1.4) with $a(x)$ and $U$ replaced by 1 and $(x-h$, $x+h)$, respectively. Then, $(3)$ is an operator in $C_{0}(R)$ which satisfies the condition in Theorem 5.2, and hence generates an $M$-semigroup in $C_{0}(R)$. If we can have (5.13) in Property III, the assumption of the vanishing at infinity for $b$ can be dropped.

6. The case of circles. Let $S$ be a circle, let $C(S)$ be the Banach space of continuous functions on $S$, and define $M$-semigroups and Lévy measures in the same manner as in $C_{0}(R)$. Suppose that we are given a finite continuous measure $s$ and a finite measure $m$ on $S$, both of which are positive for nonvoid open sets. Let $\Gamma$ be the discontinuity set for $m$. All the results in the preceding sections are carried over to this set-up. The situation is even simpler, since the state space $S$ is compact and we do not need any consideration relating to the boundary. Thus, any choice of $m$ and $s$ satisfies Property I. Also, Properties II and III are meaningless. For distinct points $x, y \in S$, we denote by $(x, y)$ the open connected set in $S$ with endpoints $x$ and $y$ such that if a point moves from $x$ to $y$ in the set $(x, y)$, it goes counterclockwise. $(x, y],[x, y)$, and $[x, y]$ are defined in like manner. The totality of nonempty open connected sets $U$ in $S$ which are different from $S$ is denoted by $\mathscr{U}$. We say that $x<y$ in $U$ if $(x, y) \subset U$. For each $U \in \mathscr{U}$, we define $\mathfrak{D}\left(D_{m} D_{s}^{+} ; U\right)$ and $D_{m} D_{s}^{+} f$ on $U$ as we did in $\S 2$. $\mathfrak{D}\left(D_{m} D_{s}^{+}\right)$is defined to be the set of $f$ such that $[f]_{U}$ belongs to $\mathfrak{D}\left(D_{m} D_{s}^{+} ; U\right)$ for every $U \in \mathbb{U}$. For each $U \in \mathbb{M}$, we define $s_{x}^{U}(y), \varphi_{x}^{U}(y)$, and $\psi_{x}^{U}(y)$ for $x, y \in U$ as follows:

$$
\begin{aligned}
& s_{x}^{U}(y)=s(x, y), 0,-s(y, x), \\
& \varphi_{x}^{U}(y)=\int_{(x, y)} s(d z) m(x, z], 0, \int_{(y, x)} s(d z) m(z, x], \\
& \psi_{x}^{U}(y)=\int_{(x, y)} s(d z) m[x, z], 0, \int_{(y, x)} s(d z) m(z, x)
\end{aligned}
$$

for $y>x, y=x$, and $y<x$, in $U$, respectively. 
We get the following theorems:

THEOREM 6.1. Let $\left\{T_{t}\right\}$ be an $M$-semigroup in $C(S)$ generated by (Y) such that $\mathfrak{T}((i)) \supset \mathfrak{T}\left(D_{m} D_{s}^{+}\right)$. Then,

(i) $\left\{T_{t}\right\}$ has Lévy measure $\left\{n_{x}(d y) ; x \in S\right\}$.

(ii) If $f \in C(S)$ and $x_{0} \notin S(f)$, then $\int_{S \backslash\{x\}} f(y) n_{x}(d y)$ is continuous at $x_{0}$.

(iii) For any $U \in \mathscr{U}$ containing $x, \int_{U \backslash\{x\}} \varphi_{x}^{U}(y) n_{x}(d y)$ or $\int_{U \backslash\{x\}}\left|s_{x}^{U}(y)\right| n_{x}(d y)$ is finite according as $x \notin \Gamma$ or $x \in \Gamma$. Moreover, we have

$$
\sup _{x \in U} \int_{U \backslash\{x\}}\left(\varphi_{x}^{U}(y)+\psi_{x}^{U}(y)\right) n_{x}(d y)<\infty
$$

for any $U \in \mathscr{U}$.

(iv) Let $U \in \mathscr{U}, f \in C(S), S(f) \subset U, x_{0} \in U$, and $f\left(x_{0}\right)=0$. If $x_{0} \notin \Gamma$, then we have

$$
\lim _{t \rightarrow 0+} t^{-1} T_{t}\left(f \varphi_{x_{0}}^{U}\right)\left(x_{0}\right)=\int_{U \backslash\left\{x_{0}\right\}} f(y) \varphi_{x_{0}}^{U}(y) n_{x_{0}}(d y) .
$$

If $x_{0} \in \Gamma$, then we have

$$
\lim _{t \rightarrow 0+} t^{-1} T_{t}\left(f s_{x_{0}}^{U}\right)\left(x_{0}\right)=\int_{U \backslash\left\{x_{0}\right\}} f(y) s_{x_{0}}^{U}(y) n_{x_{0}}(d y) .
$$

THEOREM 6.2. Let $\left\{T_{t}\right\}$ be an $M$-semigroup in $C(S)$ with infinitesimal generator (S) such that $\mathfrak{D}\left(D_{m} D_{s}^{+}\right) \subset \mathfrak{D}($ (B) $)$. Then,

(i) For each $U \in \mathscr{U}$ there are unique functions $a(x), b(x)$, and $c(x)$ on $U \backslash \Gamma$ such that, for every $f \in \mathfrak{D}\left(D_{m} D_{s}^{+}\right)$and $x \in U \backslash \Gamma$, we have

$$
\text { (S) } \begin{aligned}
f(x)= & a(x) D_{m} D_{s}^{+} f(x)+b(x) D_{s} f(x)+c(x) f(x) \\
& +\int_{S \backslash\{x\}}\left[f(y)-f(x)-\chi_{U}(y) s_{x}^{U}(y) D_{s} f(x)\right] n_{x}(d y) .
\end{aligned}
$$

$a$ and $c$ are independent of $U . a$ is bounded measurable, $c$ is continuous $\left({ }^{13}\right)$, and they satisfy (1.5). $b$ is measurable and bounded on any compact set in $U$.

(ii) For each $x \in \Gamma$ there are unique $a^{+}(x), a^{-}(x)$, and $c(x)$ such that

$$
\text { (S) } \begin{aligned}
f(x)= & (m\{x\})^{-1}\left(a^{+}(x) D_{s}^{+} f(x)-a^{-}(x) D_{s}^{-} f(x)\right)+c(x) f(x) \\
& +\int_{S \mid\{x\}}[f(y)-f(x)] n_{x}(d y)
\end{aligned}
$$

for all $f \in \mathfrak{D}\left(D_{m} D_{s}^{+}\right) . a^{+}, a^{-}$, and $c$ are bounded and satisfy (1.7).

THEOREM 6.3. Let $\alpha=\sup _{x \in S} m\{x\}$. Let (os be an operator in $C(S)$ with domain $\mathfrak{D}(\mathfrak{S})=\mathfrak{D}\left(D_{m} D_{s}^{+}\right)$such that, for each $x \in S$, there are a measure $n_{x}(d y)$ on $S \backslash\{x\}$ and a set $U_{x} \in \mathscr{U}$ containing $x$ satisfying the two conditions below. Then, (s) generates an $M$-semigroup in $C(S)$.

(13) Actually, c, defined on $R \backslash \Gamma$ in (i) and on $\Gamma$ in (ii), is continuous on $R$. 
(i) There are functions $b$ and $c$ on $S \backslash \Gamma$ such that (6.1) holds on $S \backslash \Gamma$ for $f \in \mathfrak{D}((3))$ with $a(x)$ replaced by 1 and $U$ replaced by $U_{x}$, and that (5.3), (5.4), and (5.5) hold with $R$ and $\varphi_{x}$ replaced by $S$ and $\varphi_{x}^{U}$, respectively.

(ii) There are functions $a^{+}, a^{-}$, and $c$ on $\Gamma$ such that (6.2) holds on $\Gamma$ for $f \in \mathfrak{D}($ (S) and that (1.7), (5.6), (5.7), and (5.8) hold with $R$ and $s_{x}$ replaced by $S$ and $S_{x}^{U_{x}}$, respectively.

THEOREM 6.4. Suppose that $m$ is continuous. Let (s) be the same as in Theorem 6.3 except that the condition (ii) is dropped and that $\Gamma=\varnothing$ and $\alpha=0$ in the condition (i). Then, (s) generates an $M$-semigroup in $C(S)$.

THEOREM 6.5. Let (B) be a dispersive operator in $C(S)$ with domain $\mathfrak{D}(\mathbb{S})=$ $\mathfrak{D}\left(D_{m} D_{s}^{+}\right)$satisfying the condition described in Theorem 5.3 with $S, s_{x}^{U}, \varphi_{x}^{U}$, and $\psi_{x}^{U}$ replacing $R, s_{x}, \varphi_{x}$, and $\psi_{x}$, respectively. Then, (SS generates an $M$-semigroup in $C(S)$.

EXAMPLE 6.1. Let $S$ be a unit circle, $\lambda(d y)$ be the Lebesgue measure (the ordinary length) on $S$, and $\theta_{x}(y)$ be the distance of $y$ from $x$ along the circle (hence $0 \leqq \theta_{x}(y)$ $\leqq \pi)$. Assume that $m$ is continuous. Let (sf $f$ be defined by (6.1) with $a(x)=1, b(x)$ continuous, $c(x)$ continuous and $\leqq 0, n_{x}(d y)=(2 \pi)^{-1}\left(1-\cos \theta_{x}(y)\right)^{-1} \lambda(d y)$, and $U=U_{x}$ where $U_{x}$ is $S$ minus a point $y$ such that $\theta_{x}(y)=\pi$. Let $U_{x}^{\delta}=\left\{y ; \theta_{x}(y)<\delta\right\}$. If we have

$$
\lim _{\delta \rightarrow 0+} \sup _{x \in S} \int_{U_{x}^{\delta}} \varphi_{x}^{U} x(y) n_{x}(d y)=0
$$

then $(S)$ is defined on $\mathfrak{D}\left(D_{m} D_{s}^{+}\right)$and generates an $M$-semigroup in $C(S)$. In fact, Theorem 6.4 applies to this case.

EXAMPLE 6.2. Let (s) be such as in the preceding example. Let us impose more restriction on $s$ and $m$ : suppose $s(d y)=\theta_{x_{0}}(y)^{\alpha} \lambda(d y), \alpha>-1$, and $m(d y)=$ $\theta_{x_{0}}(y)^{\beta} \lambda(d y), \beta>-1, x_{0}$ being a fixed point of $S$. Then, ${ }^{S}$ is defined on $\mathfrak{D}\left(D_{m} D_{s}^{+}\right)$ and generates an $M$-semigroup in $C(S)$ if and only if $\alpha+\beta>-1$. In fact, we have

$$
k_{1} \theta_{x}(y)^{-2} \lambda(d y) \leqq n_{x}(d y) \leqq k_{2} \theta_{x}(y)^{-2} \lambda(d y)
$$

for some positive constants $k_{1}$ and $k_{2}$, and if $\mathscr{B}$ is defined on $\mathfrak{D}\left(D_{m} D_{s}^{+}\right)$and generates an $M$-semigroup, then we have $\alpha+\beta>-1$ by virtue of Theorem 6.1 (iii), noting that $\varphi_{x}^{U} x(y)=$ const $\times \theta_{x}(y)^{\alpha+\beta+2}$ for $x=x_{0}$. It can be proved that there is a constant $k_{\alpha, \beta}$ such that $\varphi_{x}^{U}(y) \leqq k_{\alpha, \beta} \theta_{x}(y)^{(\alpha+\beta+2) \wedge 2}$ for all $x$ and $y$. Hence, conversely, if $\alpha+\beta>-1$ holds, then (6.3) is proved by (6.4) and (5) is defined and generates an $M$-semigroup.

Example 6.3. Again let (s) be the same as in Example 6.1, $m$ being continuous. Suppose $s(d y)=\lambda(d y)$. If $m$ satisfies

$$
\lim _{\delta \rightarrow 0+} \int_{U_{x}^{\delta}}\left|\log \theta_{x}(y)\right| m(d y)=0
$$


uniformly on $S$, then $\mathscr{S S}$ is defined on $\mathfrak{T}\left(D_{m} D_{s}^{+}\right)$and generates an $M$-semigroup. In fact, we have $\varphi_{x}^{U} x(y)=\int_{(x, y)} \theta_{y}(z) m(d z)$ or $\int_{(y, x)} \theta_{y}(z) m(d z)$ according as $x<y$ or $y<x$ in $U$ respectively, and hence,

$$
\int_{U_{x}^{\delta}} \varphi_{x}^{U} x(y) \theta_{x}(y)^{-2} \lambda(d y)=\int_{U_{x}^{\delta}}\left(\log \delta-\log \theta_{x}(y)+\delta^{-1} \theta_{x}(y)-1\right) m(d y)
$$

which tends to 0 as $\delta \rightarrow 0$ uniformly on $S$. Thus we have (6.3) by using (6.4), and Example 6.1 applies. Conversely, if $\left(\mathfrak{S}\right.$ is defined on $\mathfrak{D}\left(D_{m} D_{s}^{+}\right)$and generates an $M$-semigroup, then we have (6.5) for each $x \in S$, because $\int_{U_{x}^{\delta}}\left|\log \theta_{x}(y)\right| m(d y)$ is finite for fixed $\delta$ by Theorem 6.1 (iii) combined with (6.4) and (6.6).

\section{REFERENCES}

1. J.-M. Bony, Ph. Courrège et P. Priouret, Sur la forme intégro-différentielle du générateur infinitésimal d'un semi-groupe de Feller sur une variété différentiable, C. R. Acad. Sci. Paris. Sér. A-B 263 (1966), A207-A210. MR 34 \#5150a.

2. W. Feller, On second order differential operators, Ann. of Math. (2) 61 (1955), 90-105. MR 16, 824.

3. - Generalized second order differential operators and their lateral conditions, Illinois J. Math. 1 (1957), 459-504. MR 19, 1052.

4. K. Gustafson and K. Sato, Some perturbation theorems for nonnegative contraction semigroups, J. Math. Soc. Japan 21 (1969), 200-204.

5. N. Ikeda and S. Watanabe, On some relations between the harmonic measure and the Lévy measure for a certain class of Markov processes, J. Math. Kyoto Univ. 2 (1962), 79-95. MR 25 \#5546.

6. K. Itô, On stochastic differential equations, Mem. Amer. Math. Soc. No. 4 (1951). MR 12, 724.

7. —, Stochastic processes, Iwanami-Shoten, Tokyo, 1957; English transl., Yale University, New Haven, Conn., mimeographed; Russian transl., Izdat. Inostr. Lit., Moscow, 1960 and 1963. MR 23 \#A2917 and MR 32 \#6539.

8. M. Motoo, Additive functionals of Markov processes, Seminar on Probability, Vol. 15, Kakurituron Seminar, 1963. (mimeographed in Japanese)

9. —_ Application of additive functionals to the boundary problem of Markov processes. Lévy's system of U-processes, Proc. Fifth Berkeley Sympos. Math. Statist. Prob. (Berkeley, Calif., 1965/66) vol. II: Contributions to Probability Theory, Part 2, Univ. of California Press, Berkeley, Calif., 1967, pp. 75-110. MR 36 \#3414.

10. K. Sato, Integration of the generalized Kolmogorov-Feller backward equations, J. Fac. Sci. Univ. Tokyo Sect. I 9 (1961), 13-27. MR 25 \#606.

11. - On the generators of non-negative contraction semi-groups in Banach lattices, J. Math. Soc. Japan 20 (1968), 423-436. MR 37 \#6798.

12. T. Ueno, The diffusion satisfying Wentzell's boundary condition and the Markov process on the boundary. I, Proc. Japan Acad. 36 (1960), 533-538. MR 26 \#1926.

13. W. von Waldenfels, Positive Halbgruppen auf einem n-dimensionalen Torus, Arch. Math. 15 (1964), 191-203. MR 29 \#3876.

14. S. Watanabe, On discontinuous additive functionals and Lévy measures of a Markov process, Japan J. Math. 34 (1964), 53-70. MR 32 \#3137. 
15. K. Yosida, An extension of Fokker-Planck's equation, Proc. Japan Acad. 25 (1949), no. 9, 1-3. MR 12, 270.

16. - Functional analysis, Die Grundlehren der math. Wissenschaften, Band 123, Academic Press, New York and Springer-Verlag, Berlin, 1965. MR 31 \#5054.

\section{TOKYO UNIVERSITY OF EDUCATION,}

TOKYO, JAPAN 